\title{
Inactivation of NKX6.3 in the stomach leads to abnormal expression of CDX2 and SOX2 required for gastric-to-intestinal transdifferentiation
}

\author{
Jung H Yoon ${ }^{1}$, Sung S Choi ${ }^{2}$, Olga Kim ${ }^{1}$, Won S Choi ${ }^{1}$, Yong K Park ${ }^{3}$, Suk W Nam ${ }^{1}$, \\ Jung Y Lee ${ }^{1}$ and Won S Park ${ }^{1}$ \\ ${ }^{1}$ Department of Pathology, College of Medicine, The Catholic University of Korea, Seoul, South Korea; \\ ${ }^{2}$ College of Pharmacy, Sahmyook University, Seoul, South Korea and ${ }^{3}$ Department of Biostatistics, College of \\ Medicine, The Catholic University of Korea, Seoul, South Korea
}

\begin{abstract}
Intestinal metaplasia in gastric mucosa is considered a preneoplastic lesion that progresses to gastric cancer. However, the molecular networks underlying this lesion formation are largely unknown. NKX6.3 is known to be an important regulator in gastric mucosal epithelial differentiation. In this study, we characterized the effects of NKX6.3 that may contribute to gastric intestinal metaplasia. NKX6.3 expression was significantly reduced in gastric mucosae with intestinal metaplasia. The mRNA expression levels of both NKX6.3 and CDX2 predicted the intestinal metaplasia risk, with an area under the receiver operating characteristic curve value of 0.9414 and 0.9971 , respectively. Notably, the NKX6.3 expression level was positively and inversely correlated with SOX2 and CDX2, respectively. In stable AGS ${ }^{\mathrm{NKX} 6.3}$ and MKN1 ${ }^{\mathrm{NKX6.3}}$ cells, NKX6.3 regulated the expression of CDX2 and SOX2 by directly binding to the promoter regions of both genes. Nuclear NKX6.3 expression was detected only in gastric epithelial cells without intestinal metaplasia. Furthermore, NKX6.3-induced TWSG1 bound to BMP4 and inhibited BMP4-binding activity to BMPR-II. These data suggest that NKX6.3 might function as a master regulator of gastric differentiation by affecting SOX2 and CDX2 expression and the NKX6.3 inactivation may result in intestinal metaplasia in gastric epithelial cells.

Modern Pathology (2016) 29, 194-208; doi:10.1038/modpathol.2015.150; published online 8 January 2016
\end{abstract}

Gastric cancer is still one of the malignancies with high incidence and mortality rates worldwide. ${ }^{1}$ The stages of the precancerous cascade for gastric adenocarcinoma are a series of histologically recognizable changes in the gastric mucosa that follow a specific sequence: non-atrophic gastritis, multifocal atrophic gastritis, intestinal metaplasia, and dysplasia. ${ }^{2-4}$ Of these, intestinal metaplasia is the replacement of gastric epithelium by epithelium displaying an intestinal phenotype with the appearance of goblet, Paneth, and absorptive cells. It is well known that Helicobacter pylori infection, high salt intake, smoking, and alcohol consumption are risk factors for gastric intestinal metaplasia. ${ }^{5-8}$ Interestingly, altered expression of Muc5ac with

Correspondence: Professor WS Park, MD, Department of Pathology, College of Medicine, The Catholic University of Korea, 505 Banpo-dong, Seocho-gu, Seoul 137-701, South Korea.

E-mail: wonsang@catholic.ac.kr

Received 5 August 2015; accepted 9 November 2015; published online 8 January 2016 the aberrant expression of Muc2 and caudal-related homologue 2 (CDX2) was detected in intestinal metaplasia of the stomach, which is characterized by the appearance of goblet cells and is considered to be a preneoplastic stage of gastric carcinogenesis. ${ }^{9-12}$ Patients with gastric intestinal metaplasia showed a six-fold increased risk of gastric cancer than did those without gastric intestinal metaplasia. ${ }^{13}$ Although much is known about the morphology and physiology of gastric intestinal metaplasia, the regulatory mechanisms that govern their intestinal differentiation still remain unclear.

It was recently reported that NKX6.3, a third member of the NKX6 subfamily of NKX genes, is a novel transcription factor required for gastric epithelial differentiation. ${ }^{14}$ In mouse embryos, NKX6.3 expression is restricted to endoderm-derived cells in the gastric antrum, pylorus, and proximal duodenum. ${ }^{15}$ As a stratified squamous epithelium extends from the esophagus to the fundus of the mouse stomach, followed by the glandular mucosa of 
the corpus and antrum, ${ }^{16}$ it is likely that NKX6.3 is expressed in the glandular mucosa of the stomach. In our previous report, we confirmed that human NKX6.3 protein is expressed throughout the entire gastric mucosa and that NKX6.3 induces the expression of gastric foveolar mucin Muc5ac at protein and mRNA levels in addition to downregulating CDX2, an intestinal-specific homeobox transcription factor. ${ }^{17}$ Here we hypothesized that NKX6.3 has an important role in the transdifferentiation of gastric epithelium to the intestinal phenotype.

Thus we sought to investigate the effects of NKX6.3 on the differentiation of gastric epithelium and on the expression of target genes involved in gastric and intestinal differentiation in NKX6.3 stable AGS and MKN1 gastric cancer cell lines as well as in 55 non-cancerous gastric mucosae. Overall, we showed that NKX6.3 may be crucial for proper gastric differentiation and the prevention of intestinal metaplasia in gastric epithelial cells.

\section{Materials and methods}

\section{Gastric Mucosa Specimens}

A total of 55 patients with sporadic gastric cancer who underwent a gastrectomy at the Chonnam National University Hwasun Hospital were included. Non-neoplastic gastric mucosae remote $(>5 \mathrm{~cm})$ from the tumor at each corresponding region were divided into two parts. One part was frozen and used for molecular studies. The other tissues were fixed in formalin and stained with hematoxylin-eosin. The gastric mucosa specimens were obtained from fundus (2 patients), body (22), and antropyloric region (31). Informed consent was provided according to the Declaration of Helsinki. Written informed consent was obtained from all subjects. The study was approved by the Institutional Review Board of The Catholic University of Korea, College of Medicine (MC15SISI0015). There was no evidence of familial cancer in any of the patients.

Histological assessment was performed independently by two pathologists. According to the updated Sydney system, ${ }^{18}$ gastritis score of 0 (absent), 1 (mild), 2 (moderate), or 3 (marked) was assigned to each of the following parameters: activity (granulocytic infiltration), inflammation (lymphocytic and plasma cell infiltration), intestinal metaplasia, and glandular atrophy. The specimens with grade $\geq 2$, histological findings of granulocyte and mononuclear cell (mainly lymphocytes and plasma cells) infiltration were diagnosed with inflammation positive. Atrophy was defined as the loss of appropriate glands, and alcian blue staining was used to identify the intestinal metaplasia. Of 55 gastric mucosae, 15 cases showed negative histology for $H$. pylori infection, atrophy, and intestinal metaplasia and were used as a control for molecular studies.

\section{Bacterial Strain and Animal Infection}

H. pylori SS1 was cultured and infection in mice is described in the Supplementary Methods.

\section{Generation of NKX6.3 Stable Cells}

Generation of NKX6.3 stable cells are described in the Supplementary Methods.

\section{Expression of Differentiation-Related Genes}

After quantification of mRNA extracted from non-cancerous gastric mucosae, cDNA was synthesized using the Reverse Transcription Kit from Roche Molecular System (Roche, Mannheim, Germany), according to the manufacturer's protocol. Procedures are described in detail in the Supplementary Methods.

\section{Chromatin Immunoprecipitation}

For assessing the NKX6.3-binding activity in the promoter region of CDX2, SOX2, and TWSG1, chromatin immunoprecipitation assays were performed using the Thermo Scientific Pierce Agarose Chromatin Immunoprecipitation Kit (Thermo Scientific Pierce, Rockford, IL, USA), as previously described. ${ }^{17}$ Procedures are described in detail in the Supplementary Methods.

\section{Effect of CagA and GKN1 on Intestinal Metaplasia}

To determine the effect of CagA and/or GKN1 on intestinal metaplasia, the genes associated with intestinal metaplasia were examined in $\mathrm{AGS}^{\text {Mock }}$, $\mathrm{AGS}^{\mathrm{NKX6.3}}$, MKN1 ${ }^{\text {Mock}}$, and MKN1 ${ }^{\text {NKX6.3 }}$ cells at time course after transfection with CagA or shGKN1 as described previously. ${ }^{19}$ The primer sequences are shown in Supplementary Table S1. The expression of $H$. pylori CagA and differentiation-associated proteins was examined by real-time PCR and western blotting analysis, as previously described. ${ }^{19}$

\section{Immunohistochemistry and Immunofluorescence Assay}

Immunohistochemical evaluation of the NKX6.3 expression in non-neoplastic gastric mucosa was carried out as described previously. ${ }^{20}$ Immunohistochemistry and immunofluorescent analysis were processed according to standard procedures as described in the Supplementary Methods.

\section{Co-Immunoprecipitation}

Cells were lysed and processed according to standard procedures as described in the Supplementary Methods. 


\section{Statistical Analysis}

Chi-Square, Pearson's and Student's $t$-tests were used to analyze the correlation of NKX6.3, SOX2, GKN1, CDX2, Muc5ac, and Muc2 expression with intestinal metaplasia, respectively. A $P$-value $<0.05$ was considered the limit of statistical significance. To further evaluate the diagnostic usefulness of the markers based on dichotomous classification, receiver operating characteristic curve analysis was used. A receiver operating characteristic curve is a plot of the true-positive fraction vs the false-positive fraction evaluated for all possible cutoff point values.

\section{Results}

\section{NKX6.3 and CDX2 Predict Gastric Intestinal Metaplasia}

We determined the expression of NKX6.3 in different regions of the human stomach and its distribution within gastric glands in 55 non-cancerous gastric mucosae. The levels of NKX6.3 mRNA expression were similar in the proximal (fundus and body) and distal (antrum and pylorus) stomach (Figure 1a). Interestingly, NKX6.3 mRNA expression was significantly reduced in both proximal and distal gastric mucosa with intestinal metaplasia (Figure 1b). NKX6.3 immunoreactivity was detected mainly in the nucleus of several types of glandular cells (Figures 1c and d). In mice, both the forestomach, lined by squamous cells, and the glandular stomach also showed immunopositivity for NKX6.3 (Figures 1e and f).

Histologically, granulocyte and mononuclear cell infiltrations, as well as atrophy and intestinal metaplasia, were found in 11 (20\%), 42 (76\%), 18 $(33 \%)$, and $20(36 \%)$ of the 55 non-neoplastic gastric mucosal tissues, respectively. Compared with 15 control gastric mucosae without $H$. pylori infection, atrophy, and intestinal metaplasia, NKX6.3 mRNA expression was decreased in $28(70 \%)$ out of 40 gastric mucosae (Figure 2a). Nineteen (95\%) of 20 gastric mucosae with intestinal metaplasia showed reduced NKX6.3 mRNA expression (Figure 2a). In addition, NKX6.3 mRNA expression was significantly reduced in cases with mononuclear cell infiltration $(P<0.0001)$, atrophy $(P=0.0274)$ and intestinal metaplasia $(P<0.0001)$ (Figure 2b) but not in cases with granulocytic infiltration $(P=0.3550) \quad$ (Figure 2c). Interestingly, NKX6.3 predicted atrophy and intestinal metaplasia risk with an area under the receiver operating characteristic curve value of 0.6892 and 0.9414 , respectively (Figure 2d).

When we compared the expression level of CDX2 with the degree of gastritis, CDX2 was also significantly increased in gastric mucosae with mononuclear cell infiltration $(P=0.0036)$, atrophy $(P=0.0038)$, and intestinal metaplasia $(P<0.0001)$ (Figure 2e). Additionally, CDX2 predicted gastric mucosal atrophy and intestinal metaplasia risk with an area under the receiver operating characteristic curve value of 0.7613 and 0.9971 , respectively (Figure 2f).

\section{H. pylori CagA Reduces NKX6.3 Expression}

We next tested the hypothesis that $H$. pylori infection is associated with a reduction in the DNA copy number for NKX6.3. As CagA(+) H. pylori SS1 has been found to consistently infect mice at a high level and establish infections that survive over many months, the SS1 strain is usually proposed as the standard for experimental infection studies. ${ }^{21}$ The effects of $H$. pylori infection on NKX6.3, CDX2, and SOX2 expression in mice were analyzed in three $H$. pylori-infected and two non-infected C57BL/6 female mice. The gastric mucosal tissues from H. pylori-infected C57BL/6 mice showed decreased DNA copy number, mRNA transcript, and protein expression for NKX6.3 (Figures 3a-c). Although H. pylori CagA did not affect the DNA copy number for CDX2 and SOX2 genes (Figure 3a), CagA increased CDX2 and decreased SOX2 expression (Figures $3 \mathrm{~b}$ and $\mathrm{c}$ ). When we investigated the association of NKX6.3 with the presence of $H$. pylori CagA, which was detected in 26 (47\%) of 55 human non-cancerous gastric mucosae by western blotting analysis, $^{22}$ the NKX6.3 DNA copy number and mRNA expression were significantly lower in CagA-positive gastric mucosa than in CagA-negative cases $(P<0.0001)$ (Figures $3 \mathrm{~d}$ and e). In addition, there was no significant difference in the DNA copy numbers for $C D X 2$ and $S O X 2$ genes between CagA-positive and -negative gastric mucosae (Figure 3d). However, CagA-positive gastric mucosae showed increased CDX2 and decreased SOX2 mRNA expression (Figure 3e). These results indicate that $H$. pylori CagA may have a role in the inactivation of NKX6.3 and the induction of CDX2.

\section{NKX6.3 Controls Differentiation in Gastric Epithelial Cells}

To determine whether NKX6.3 contributes to the differentiation of human gastric epithelial cells, we investigated the association of NKX6.3 with the expression of gastric markers, including SOX2 and GKN1, and the intestinal marker, CDX2. As shown in Figure 4, NKX6.3 mRNA expression showed an inverse relationship with the expression of $C D X 2$ (Figure 4a) and a positive correlation with $S O X 2$ and GKN1 expression in gastric mucosal tissues (Figures $4 \mathrm{~b}$ and c) $(P=0.0001)$. In addition, SOX2 expression was positively correlated with that of GKN1 $(P<0.0001)$, whereas $C D X 2$ was inversely related to $S O X 2$ and GKN1 expression (Figures $4 \mathrm{~d}-\mathrm{f}$ ) $(P<0.0001)$, suggesting that NKX6.3 may control the differentiation of gastric epithelial cells. 
a

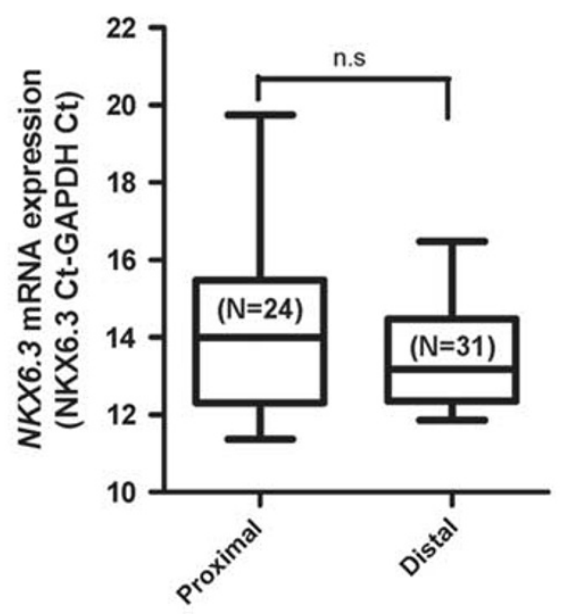

Site
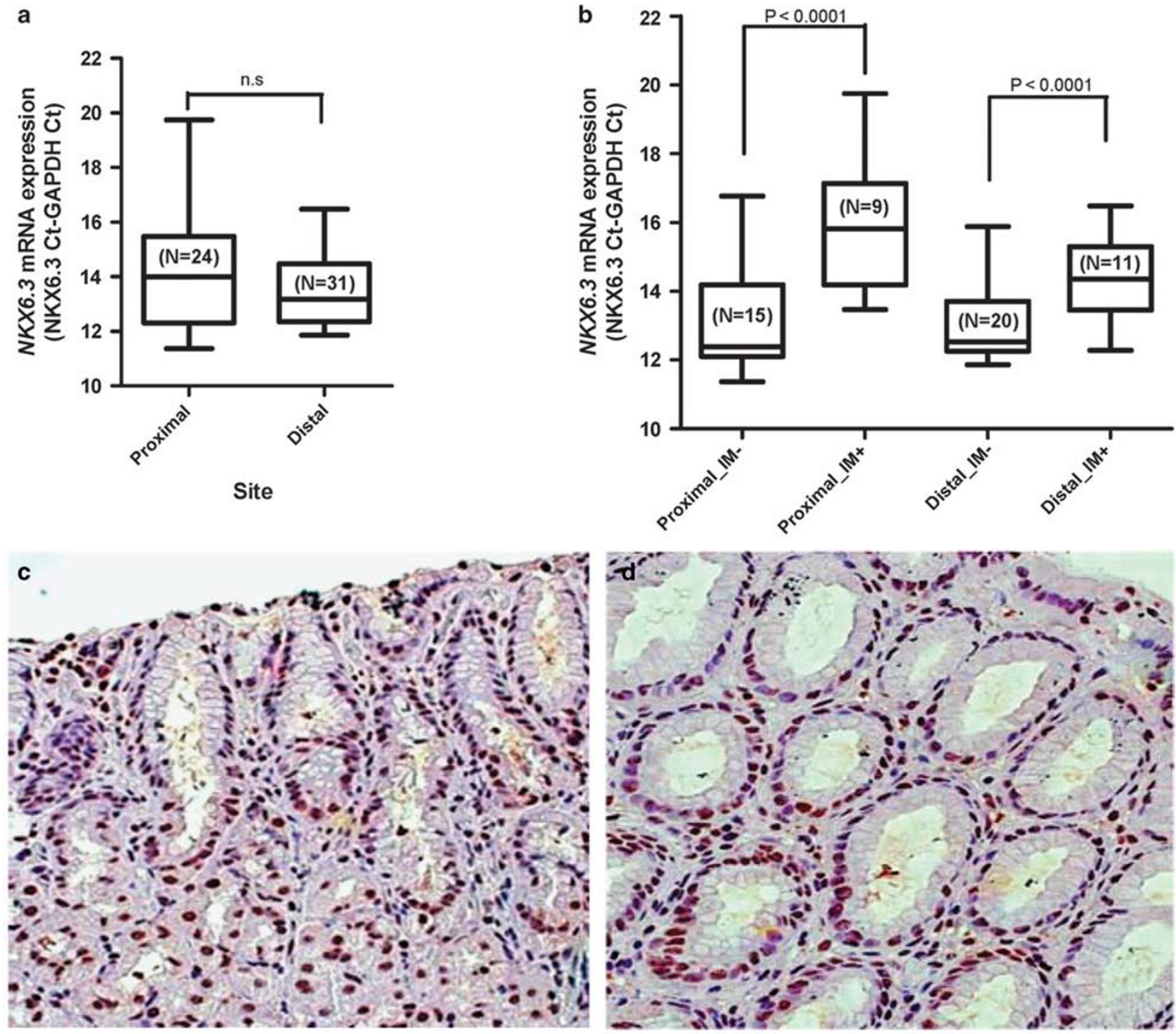

e.

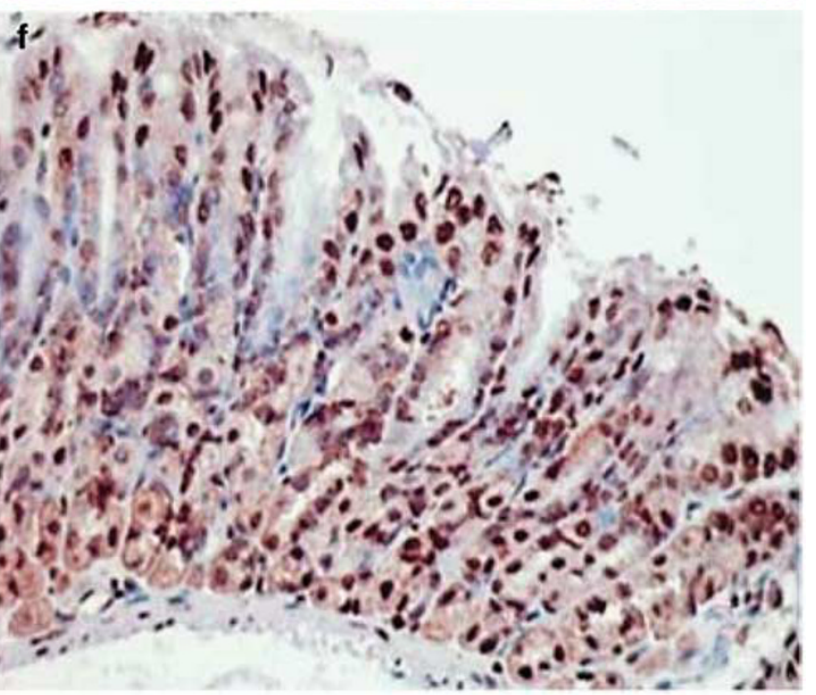

Figure 1 Expression and localization of NKX6.3 in the stomach. (a) NKX6.3 mRNA expression in the 24 proximal and 31 distal stomach. (b) Of these, proximal and distal stomach with intestinal metaplasia showed significantly reduced NKX6.3 mRNA expression. IM, intestinal metaplasia. (c and d) Intense nuclear NKX6.3 staining was detected in glandular epithelia in the body (c) and pylorus (d) of human stomach. (e and f) In mice, nuclear NKX6.3 staining was also observed in the forestomach, lined by squamous cells (e), and in the glandular stomach (f). 
Based on these observations, we performed chromatin immunoprecipitation assays followed by PCR and real-time PCR to identify the NKX6.3binding site within promoter upstream sequences of the CDX2 and SOX2 genes in AGS ${ }^{\text {Mock }}$ and MKN1 ${ }^{\text {Mock }}$ cells as well as in AGS $^{\text {NKX6.3 }}$ and MKN1 ${ }^{\mathrm{NKX} 6.3}$ cells. We defined the region upstream of the genes (between -6 and $+1 \mathrm{~kb}$ ), which overlapped with the transcription start site designated as $0 \mathrm{~kb}$. NKX6.3 showed binding activity from -6 to $+1 \mathrm{~kb}$ relative to the CDX2 and $S O X 2$ transcription start site in AGS (Figures 5a-d) and MKN1 (Supplementary Figure S1) gastric cancer cells. In particular, NKX6.3 occupancy was 7.2-fold enriched at -4376 to $-4215 \mathrm{bp}$ from the transcription start site of CDX2 gene and 4.75-fold enriched at -3051 to $-2838 \mathrm{bp}$ from the transcription start site of SOX2 (Figures 5c and d). In NKX6.3 stable cells, NKX6.3 induced SOX2 and Muc5ac mRNA expression but reduced $C D X 2$ and Muc2 expression (Figures 5e-h). In addition, NKX6.3 also increased the expression of SOX2 proteins and decreased CDX2 protein expression at 4 days after culture (Figure 5i). However, NKX6.3 silencing with shNKX6.3 induced CDX2 and Muc2 expression but reduced SOX2 and Muc5ac expression (Figures 5ei), indicating that NKX6.3 may control the differentiation of gastric mucosal epithelial cells by regulating gastric and intestinal markers.

\section{Loss of NKX6.3 Expression Is Sufficient for Gastric Intestinal Metaplasia}

Next we examined the expression levels of NKX6.3, SOX2, GKN1, CDX2, Muc5ac, and Muc2 in human gastric mucosa using immunofluorescent assays. As expected, NKX6.3 was abundant in the nuclei of gastric epithelial cells without intestinal metaplasia (Figures 6a and b), and the expression of GKN1, SOX2, and Muc5ac was mainly detected in portions of these NKX6.3-positive cells (Figures 6b-d). In regions of gastric intestinal metaplasia, both CDX2 and Muc2 proteins were strongly expressed in the gastric epithelium lacking NKX6.3, SOX2, Muc5ac, and GKN1 expression (Figures 6a-e).

\section{H. pylori CagA Induces Gastric Intestinal Metaplasia by Downregulating SOX2 and Upregulating CDX2}

As $H$. pylori infection is known to contribute to CDX2 expression and induce intestinal metaplasia, ${ }^{23}$ we investigated the effects of CagA on gastric intestinal metaplasia in AGS and MKN1 gastric cancer cell lines. Ectopic expression of CagA downregulated NKX6.3, GKN1, and SOX2 mRNA expression (Figures 7a-c) and upregulated CDX2 expression in both AGS and MKN1 cells in a timedependent manner (Figure 7d). These results were confirmed in CagA-transfected AGS Mock, MKN1 ${ }^{\text {Mock, }}$, $\mathrm{AGS}^{\mathrm{NKX} 6.3}$, and MKN1 ${ }^{\mathrm{NKX} 6.3}$ cells by western blotting analysis (Figure 7e). In addition, ectopic expression of CagA induced the expression of bone morphogenic protein 4 (BMP4) and p-SMAD1/5/8 proteins, whereas NKX6.3 reduced the expression of p-SMAD1/5/8. However, NKX6.3 did not affect BMP4 protein expression (Figure 7e). These results suggest that CagA-positive $H$. pylori infection leads to increased CDX2 and p-SMAD1/5/8 expression through attenuated expression of NKX6.3 at mRNA and protein levels.

\section{NKX6.3 Inhibits BMP4 Signaling by Inducing TWSG1}

Activation of the $\mathrm{BMP} / \mathrm{p}-\mathrm{SMAD}$ pathway leads to the expression of CDX2 and has a crucial role in the development of intestinal metaplasia. ${ }^{24,25}$ As NKX6.3 did not affect BMP4 expression, we examined the effects of NKX6.3 on TWSG1, which is a novel inhibitor for the BMP signaling pathway ${ }^{26,27}$ in AGS and MKN1 cells. Treatment with BMP4 induced p-SMAD1/5/8 and CDX2 expression and downregulated SOX2 expression in AGS and MKN1 cells (Figure 8a). However, it had no effect in NKX6.3 stable AGS ${ }^{\text {NKX6.3 }}$ and MKN1 ${ }^{\text {NKX6.3 }}$ cells (Figure 8a).

Next we investigated whether NKX6.3 is involved in the regulation of TWSG1 expression. In NKX6.3 stable cells, NKX6.3 induced TWSG1 RNA and protein expression by directly binding to promoter upstream sequences ( -2165 to $-1916 \mathrm{bp}$ and -1758 to $-1463 \mathrm{bp}$ ) from transcription start site of the

\footnotetext{
Figure 2 NKX6.3 expression in gastric mucosae. (a) In real-time PCR assay, reduced expression of NKX6.3 mRNA was found in 28 (51\%) of the 55 gastric mucosae. The mean value of mRNA expression in gastric mucosae without $H$. pylori infection, atrophy, and intestinal metaplasia was used as a control. mRNA expression change in each case was further normalized to the mean value of those in control gastric mucosae and reduced NKX6.3 expression was defined as < 0.5 fold change. (b) In scatter plot analysis, NKX6.3 mRNA expression was closely associated with mononuclear cell infiltration (Student's $t$-test, $P=0.0001)$, atrophy $(P=0.0274)$, and intestinal metaplasia $(P<0.0001)$ but not with granulocytic infiltration $(P=0.3550)$. (c) Receiver operating characteristic curve analysis using NKX6.3 to distinguish patients with atrophy and intestinal metaplasia from human gastric mucosal tissues. NKX6.3 yielded an area under the curve (AUC) value of 0.6892 (95\% confidence interval (CI), 0.5463-0.8321; $P=0.02386$ ) and 0.9414 (95\% CI, 0.8847-0.9981; $P<0.0001)$ in distinguishing atrophy and intestinal metaplasia, respectively. (d) Scatter plot analysis showed that CDX2 mRNA expression was closely associated with mononuclear cell infiltration (Student's $t$-test, $P=0.0038)$, atrophy $(P=0.0038)$ and intestinal metaplasia $(P<0.0001)$ but not with granulocytic infiltration $(P=0.9173)$. MNC, mononuclear cell; IM, intestinal metaplasia; PMN, polymorphonuclear leukocytes. (e) Receiver operating characteristic curve analysis using CDX2 to distinguish patients with atrophy and intestinal metaplasia. CDX2 yielded an AUC value of 0.7613 (95\% CI, 0.6250-0.8976; $P=0.0018$ ) and 0.9971 (95\% CI, 0.9895-1.005; $P<0.0001)$ in distinguishing atrophy and intestinal metaplasia, respectively. IM, intestinal metaplasia; MNC, mononuclear cell; PMN, polymorphonuclear leukocytes.
} 

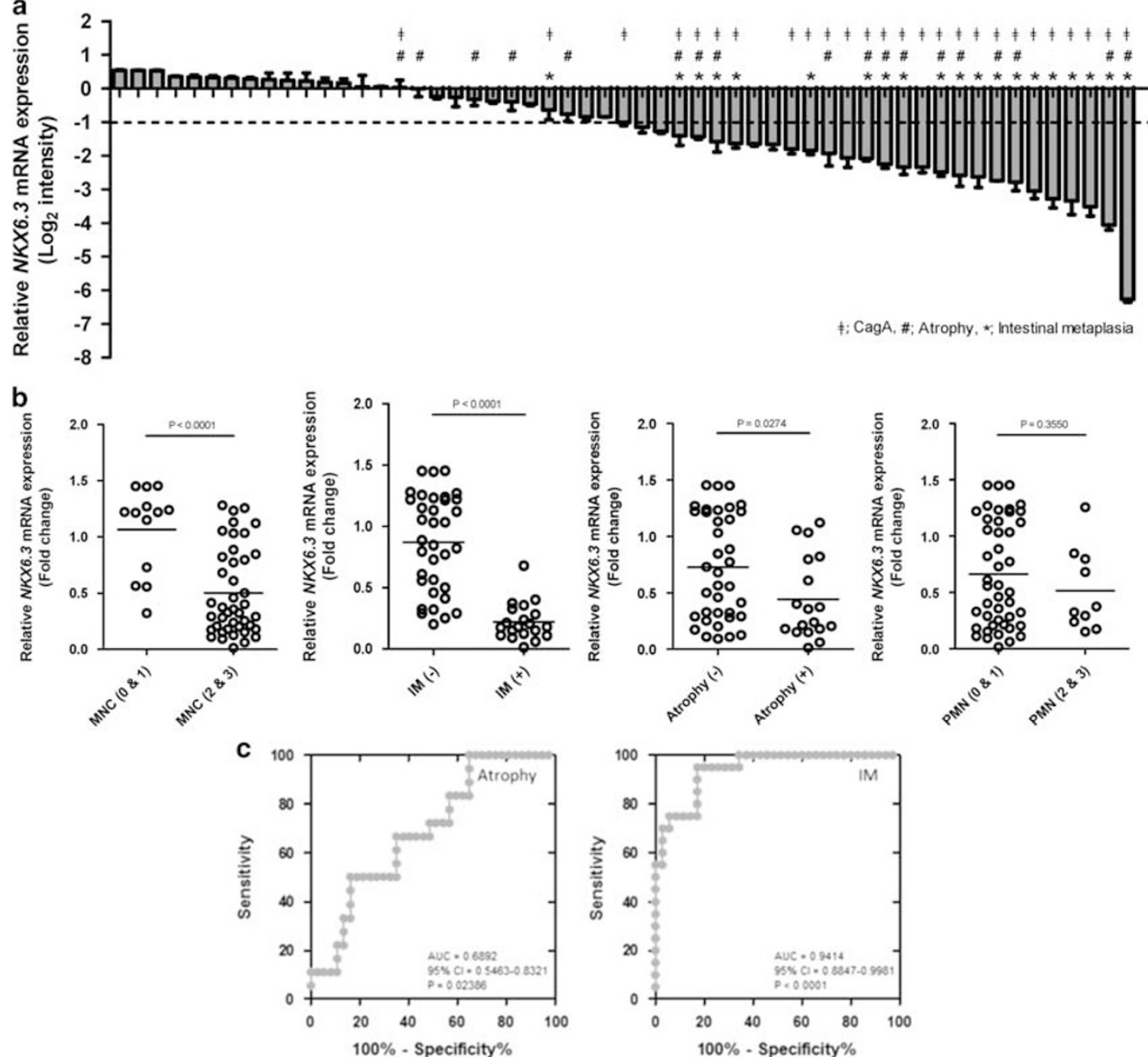

d
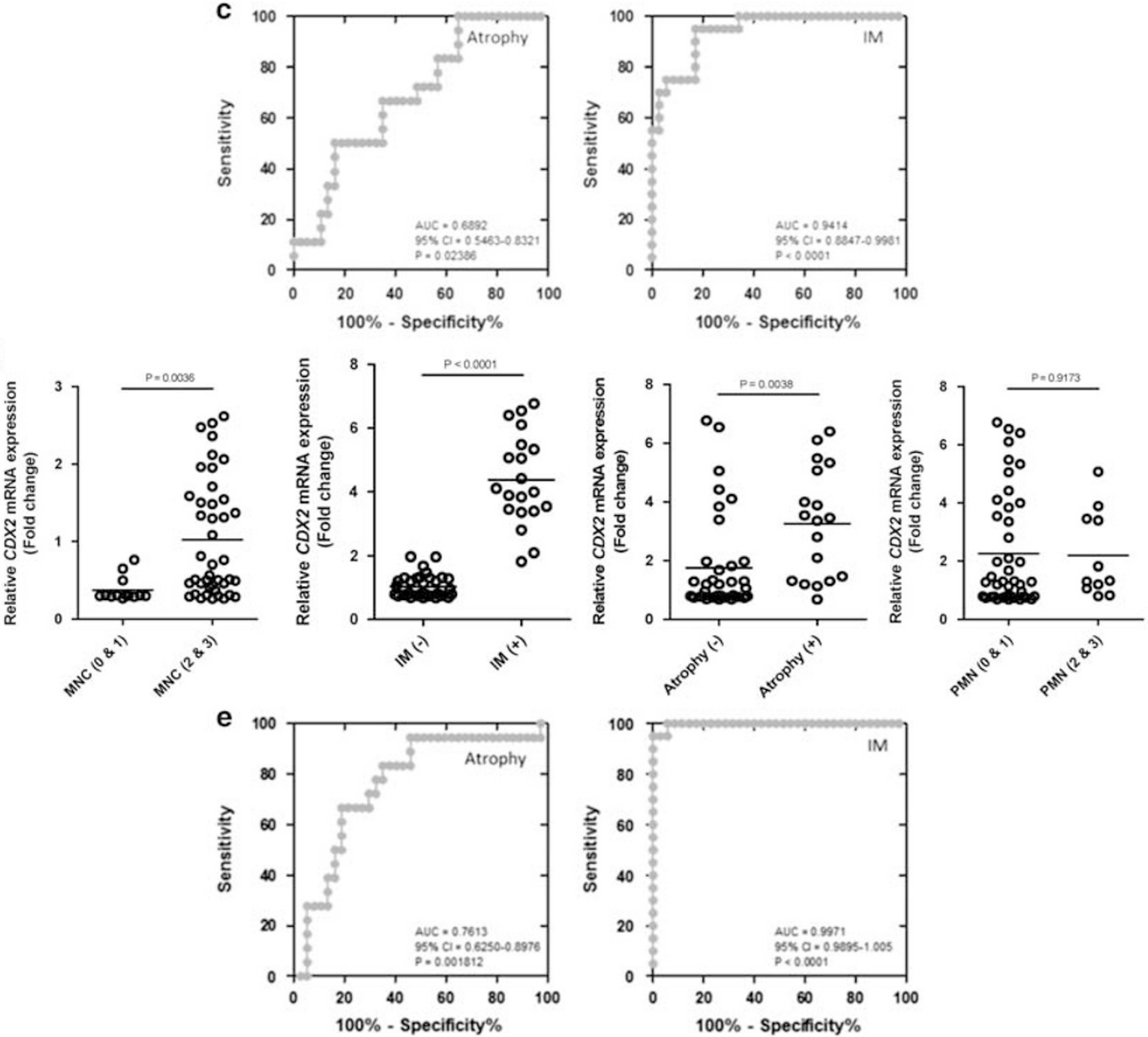
TWSG1 gene (Figures 8b and c). However, NKX6.3 did not affect bone morphogenic protein type II receptor (BMPR-II) protein expression (Figure 8b). In the immunoprecipitation assay, NKX6.3-induced TWSG1 bound directly to BMP4 and inhibited BMP4-binding activity to BMPR-II in AGS and MKN1 cells, whereas silencing NKX6.3 or TWSG1 recovered BMP4 binding to BMPR-II (Figure 8d). These results suggest that NKX6.3 may inhibit gastric intestinal metaplasia by inactivating the $\mathrm{BMP} / \mathrm{p}-\mathrm{SMAD}$ pathway through upregulation of TWSG1 expression.

\section{NKX6.3 Controls Gastric Differentiation in a GKN1-Independent Manner}

Previously, we reported that GKN1 inhibits the carcinogenic potential of CagA by direct binding to CagA. ${ }^{19}$ Thus, to determine whether NKX6.3-induced gastric differentiation is dependent on GKN1, we examined the NKX6.3, CDX2, and SOX2 expression in $\mathrm{AGS}^{\mathrm{NKX6.3}}$ and MKN1 ${ }^{\mathrm{NKX6.3}}$ cells after GKN1 silencing with shGKN1 (Supplementary Figure S2a). Notably, GKN1 silencing did not affect the expression levels of NKX6.3, SOX2, and CDX2 at the mRNA level (Supplementary Figures S2b-d), suggesting that NKX6.3 may regulate gastric differentiation in a GKN1-independent manner.

\section{Discussion}

The gastric epithelium is a simple columnar epithelium composed of gastric pits and gastric glands. Gastric pits are lined by surface mucous cells, whereas gastric glands are composed of various kinds of cells, including mucous neck cells, parietal cells, chief cells, and enteroendocrine cells. ${ }^{28}$ The generation of surface mucous cells, which takes 3 days, is regulated by Indian hedgehog and epidermal growth factor. ${ }^{28-31}$ It was recently suggested that several differentiation-associated genes such as NKX6.3, SOX2, CDX2, PDX1, and OCT1 have an important role in gastric differentiation and intestinal metaplastic formation. ${ }^{32}$ SOX2 contributes to the development of foregut-derived organs, such as the esophagus and stomach, ${ }^{33,34}$ and controls the expression of pepsinogen A and Muc5ac. ${ }^{35,36}$ The expression of SOX2 is downregulated during the progression from incomplete to complete intestinal metaplasia. ${ }^{37}$ In addition, it was suggested that SOX2 can function as a tumor suppressor by regulating the cell cycle and apoptosis. ${ }^{38}$ In particular, CDX2 is an initiator gene involved in the embryonic development and axial patterning of the alimentary tract as well as maintenance of the adult intestinal epithelium by functioning as a transcription factor for Muc2, lactase, intestinal alkaline phosphatase, and furin. ${ }^{32,39-41}$

NKX6.3 is identified as a selective regulator of $\mathrm{G}$ - and D-cell lineages in the antrum/pylorus of mice stomach. ${ }^{14}$ However, the function and expression pattern of human NKX6.3 protein still remain unknown. Here we found that NKX6.3 mRNA expression levels were similar in the human proximal (fundus and body) and distal (antum and pylorus) stomach and was reduced in gastric mucosa with intestinal metaplasia (Figures $1 \mathrm{a}$ and b). NKX6.3 expression was detected mainly in the nuclei of several types of glandular epithelia (Figures 1c and d). In mice, both the forestomach, lined by squamous cells, and the glandular stomach also showed immunoreactivity against NKX6.3 (Figures 1e and f). These data suggest that NKX6.3 may have an important role in the entire gastric mucosal epithelia of humans and mice. Interestingly, reduced NKX6.3 expression was found in 28 (51\%) of the 55 gastric mucosae (Figure 2a), and the expression levels were closely correlated with mononuclear cell infiltration $(P<0.0001)$, atrophy $(P=0.0274)$, and intestinal metaplasia $(P<0.0001)$ (Figure 2b). In keeping with the published reports of CDX2 expression in gastric intestinal metaplasia, ${ }^{40,42}$ CDX2 expression was markedly increased in gastric mucosae with intestinal metaplasia $(P<0.0001)$ and its expression was also associated with mononuclear cell infiltration $(P=0.0036)$ and atrophy $(P=0.0038)$ (Figure 2d). When the ability of NKX6.3 to discriminate patients with atrophy and intestinal metaplasia was analyzed using a receiver operating characteristic curve, NKX6.3 predicted gastric mucosal atrophy and intestinal metaplasia risk with an area under the curve value of 0.6892 and 0.9414 , respectively (Figure 2c). In addition, CDX2 predicted atrophy and intestinal metaplasia risk, with area under the curve values of 0.7613 and 0.9971 , respectively (Figure 2e). Thus our data implicate NKX6.3 and CDX2 as important in gastric mucosal inflammation and as candidate biomarkers for atrophy and intestinal metaplasia in the gastric mucosa.

Because $H$. pylori causes DNA damage and chromosomal aberrations in gastric epithelial cells, ${ }^{43,44}$ we set out to determine whether $H$. pylori CagA is involved in the expression of NKX6.3, CDX2, and SOX2 genes. In the gastric mucosae of humans and mice, CagA-positive $H$. pylori infection was negatively associated with NKX6.3 DNA copy number as well as with mRNA and protein expression (Figures 3a-e). In addition, $H$. pylori CagA induced CDX2 mRNA expression and suppressed SOX2 expression in the gastric mucosae of both humans and mice (Figure 3e). Although we could not completely rule out the possibility of underestimating the prevalence of genetic and epigenetic alterations to NKX6.3, these data suggest that CagA-positive $H$. pylori infection may be a driving event behind the inactivation of NKX6.3 and the induction of CDX2 in gastric mucosae.

Notably, NKX6.3 mRNA expression showed an inverse relationship with the expression of CDX2 (Figure 4a) and a positive correlation with $S O X 2$ and 

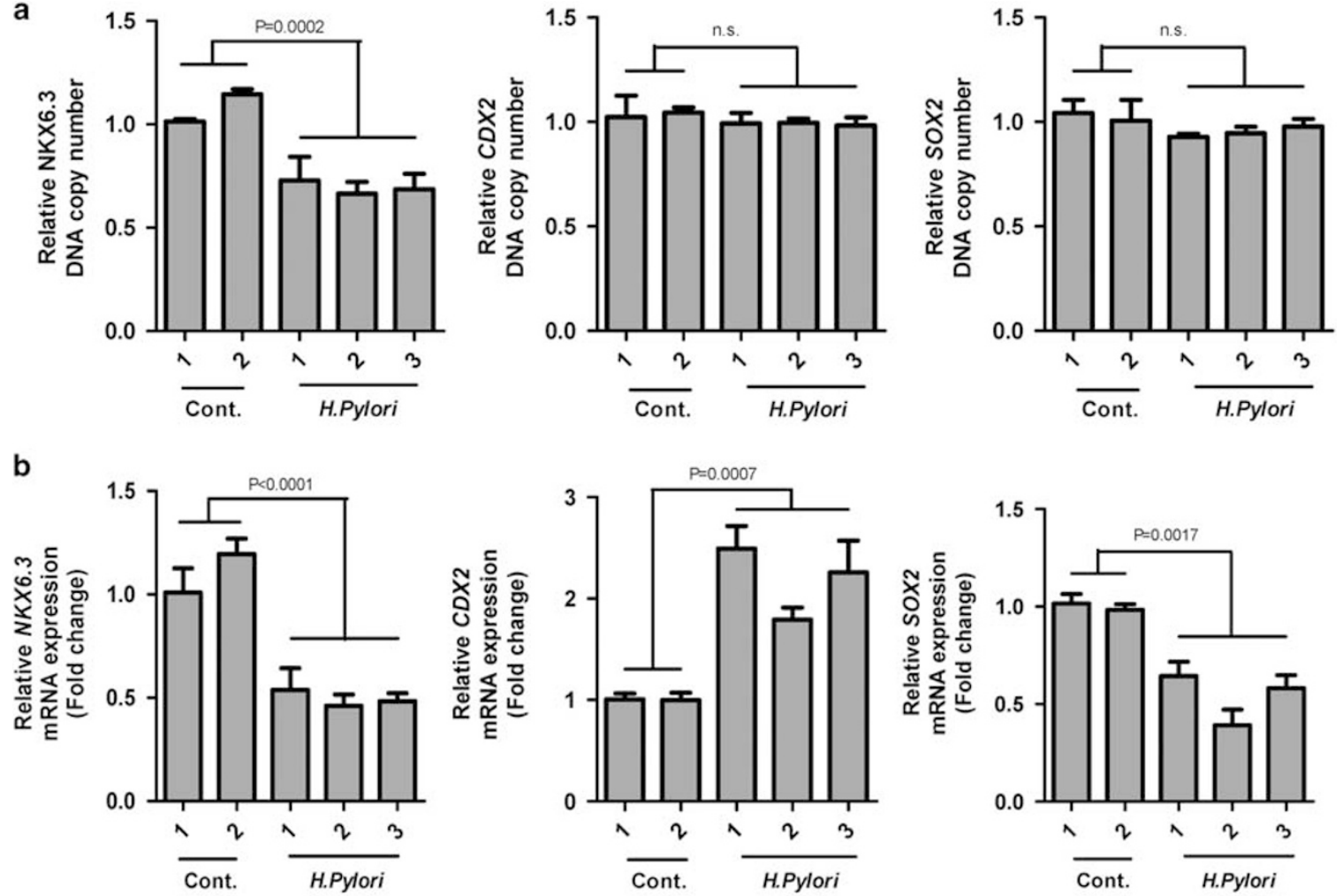

C
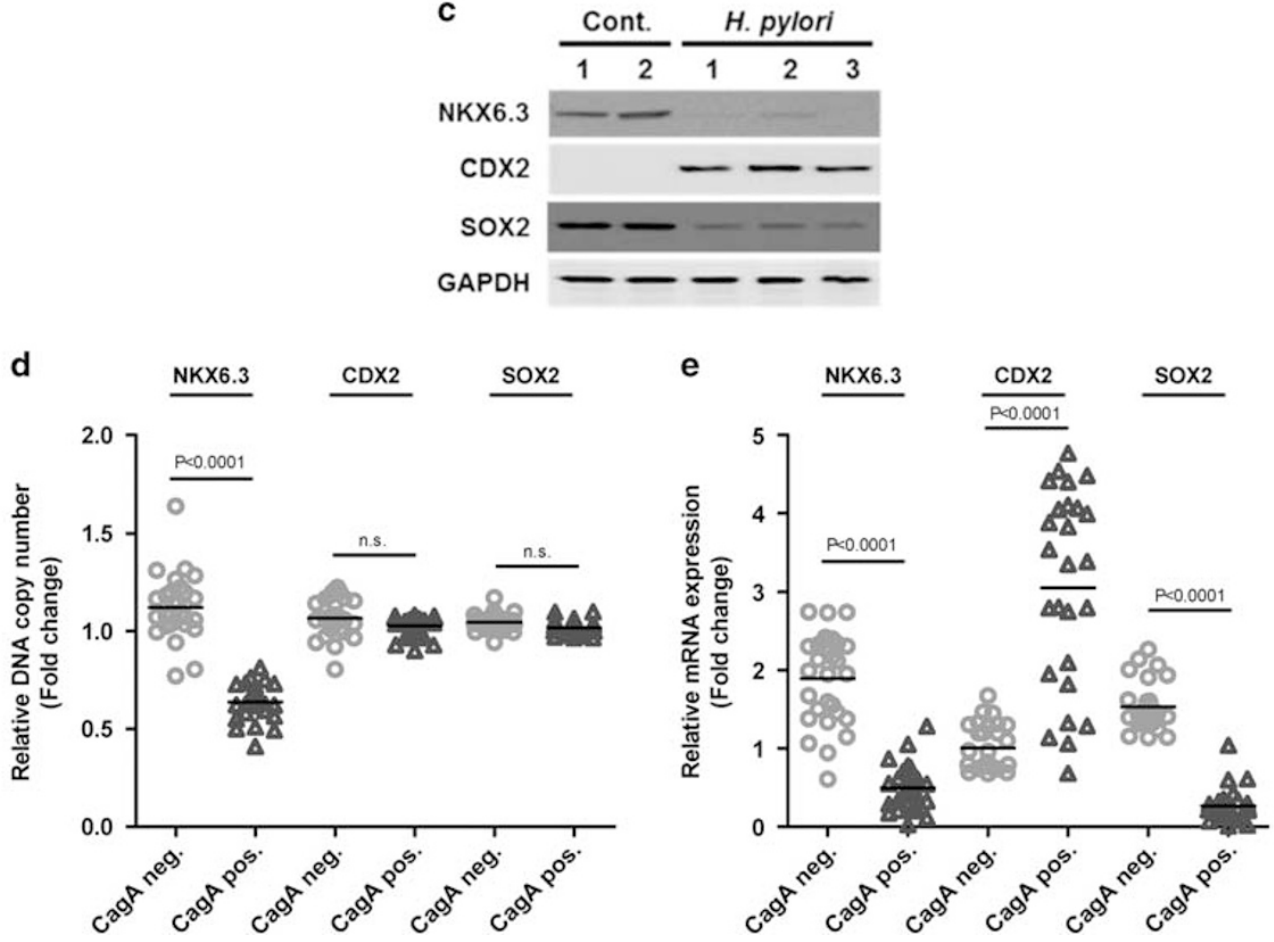

Figure 3 Effects of $H$. pylori on NKX6.3 expression. (a-c) Gastric mucosae from mice infected with CagA (+) H. pylori showed decreased NKX6.3 DNA copy number (a), mRNA transcript (b), and protein expression (c), compared with those of control (cont.). In addition, H. pylori infection decreased SOX2 mRNA expression and increased CDX2 mRNA expression. However, H. pylori infection did not affect the DNA copy numbers of SOX2 and CDX2. (d and e) Scatter plot illustrates that NKX6.3, CDX2 and SOX2 DNA copy number (d) and mRNA expression level (e) were significantly lower in H. pylori CagA-positive (pos.) than in CagA-negative (neg.) human non-cancerous gastric mucosal tissues $(P<0.01)$, but there was no significant differences in the DNA copy number of $C D X 2$ and $S O X 2$ genes. 

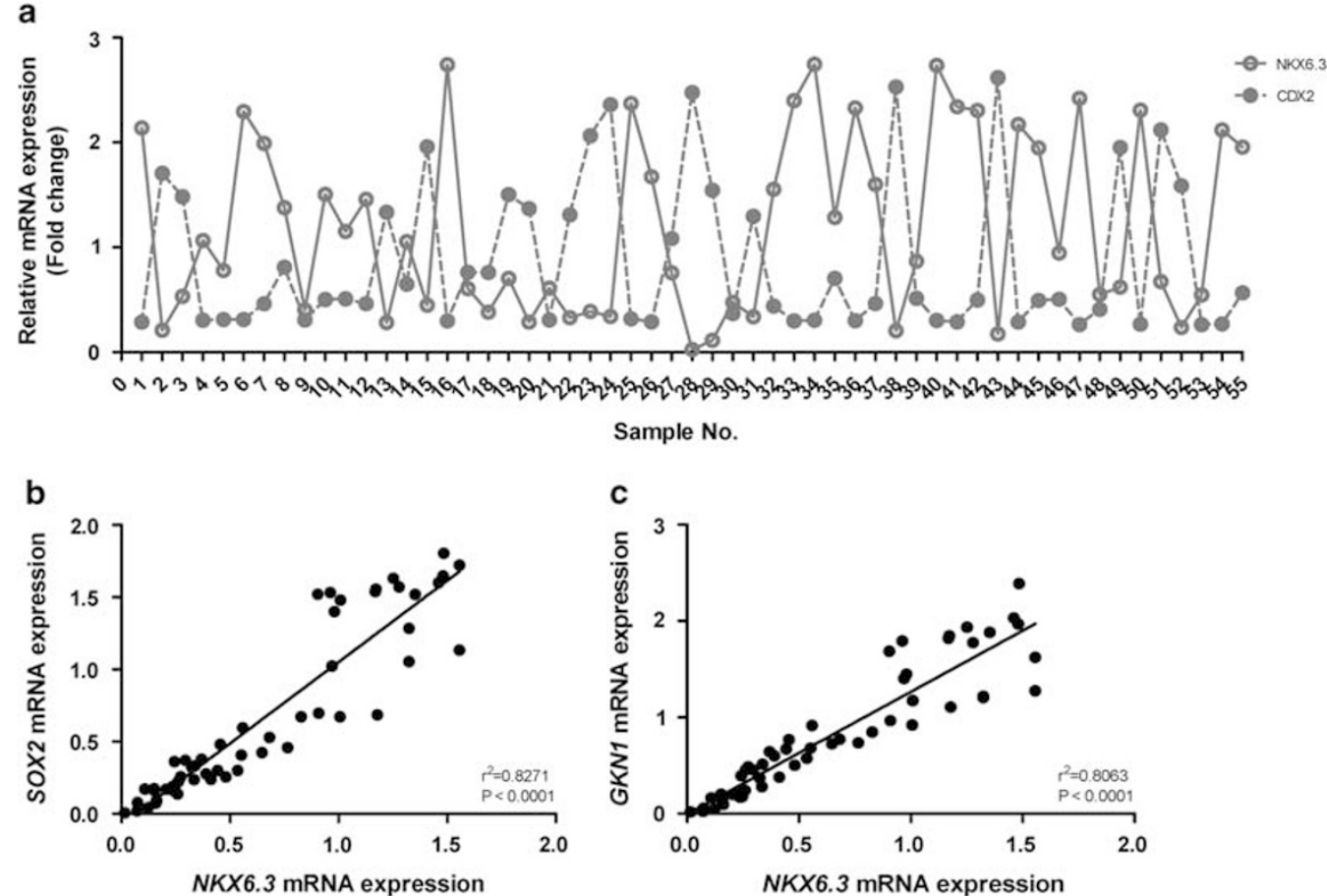

Sample No.
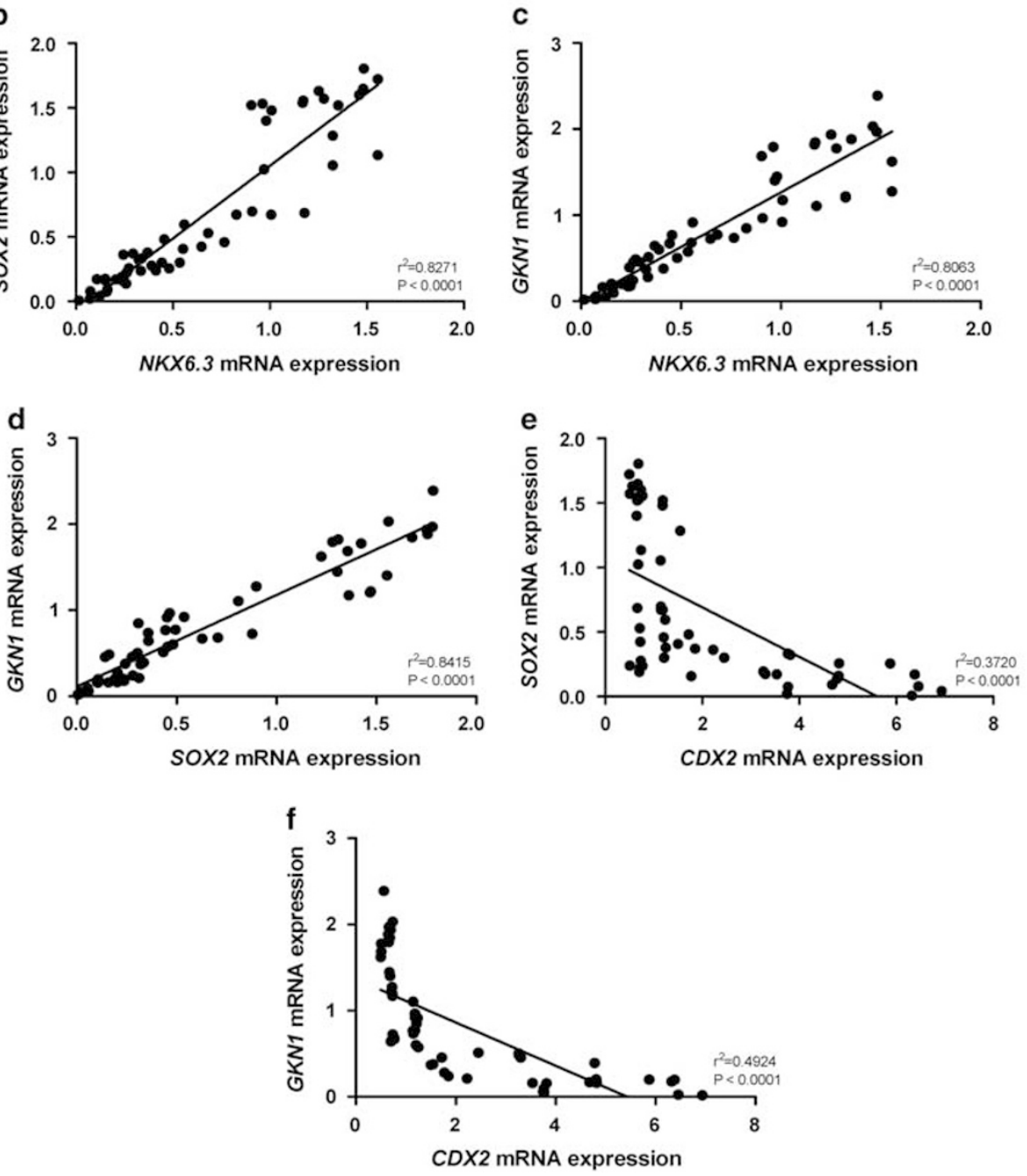

Figure 4 NKX6.3 expression was closely associated with the expression of gastric and intestinal markers. (a) NKX6.3 mRNA expression showed an inverse correlation with the expression of CDX2. (b and c) A positive correlation of NKX6.3 expression with SOX2 (b) and GKN1 (c) was observed in 55 gastric mucosae (Pearson's test, $P<0.0001$ ). (d) There was a positive correlation between GKN1 and SOX2 expression $(P<0.0001)$. (e and $\mathbf{f})$ An inverse association of $C D X 2$ expression with $S O X 2$ and GKN1 expression levels was found $(P<0.0001)$.

GKN1 expression in gastric mucosal tissues (Figures $4 \mathrm{~b}$ and c). Expectedly, SOX2 expression was positively correlated with GKN1 (Figure 4d), whereas CDX2 expression was inversely correlated with SOX2 and GKN1 (Figures 4e and f). These results are in line with reports that there was a significant inverse correlation between the expression of SOX2 and CDX2 in gastric adenocarcinomas, ${ }^{45}$ as well as reports that Lgr5-positive intestinal stem cells require CDX2 to maintain their intestinal identity and are converted into pyloric stem cells in the absence of CDX2 ${ }^{46}$ Although further studies on larger populations are necessary to clarify these initial observations, our data suggest 
a

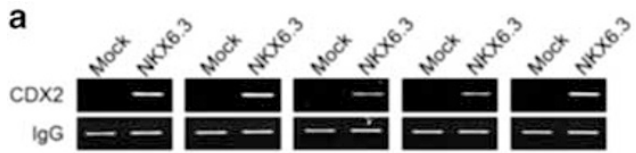

C

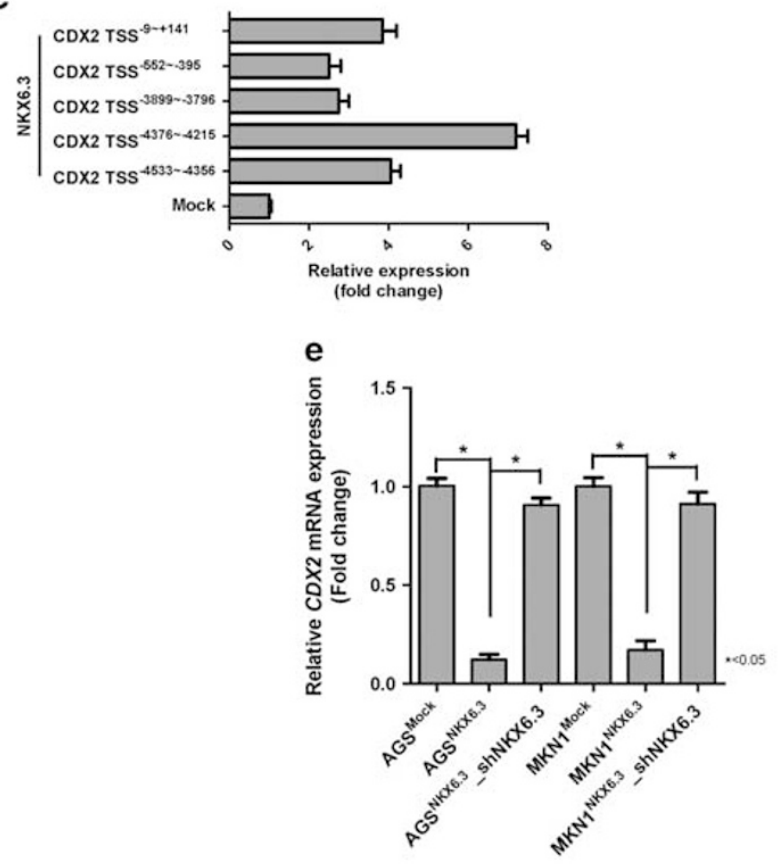

g

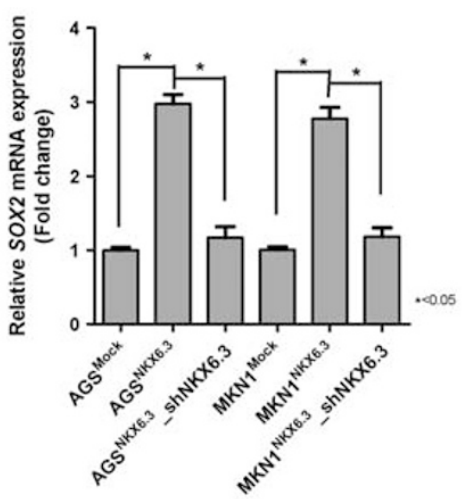

b

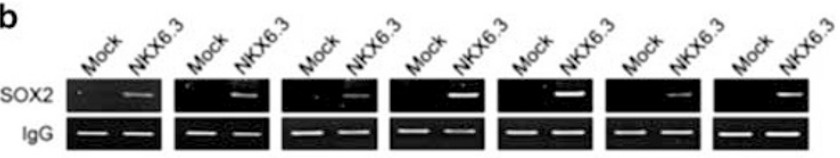

d
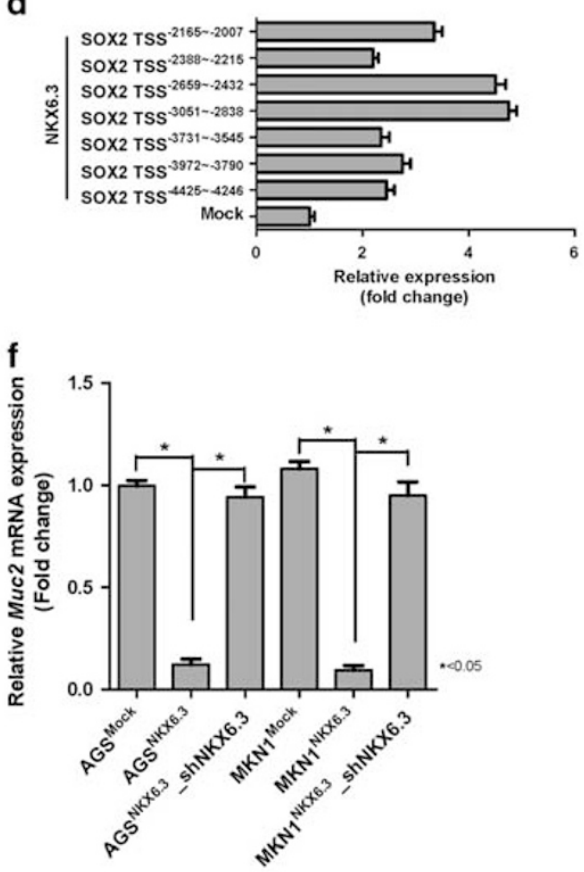

h

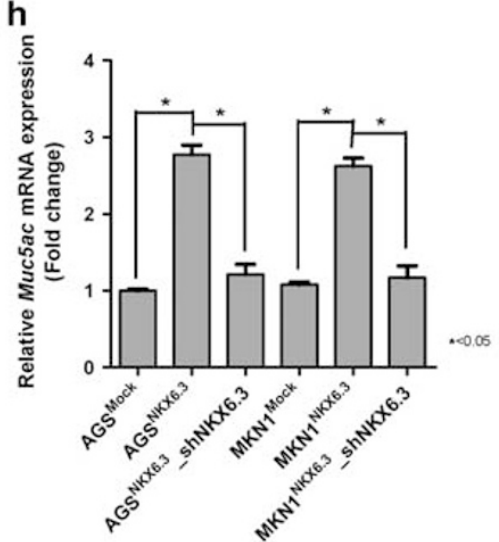

i

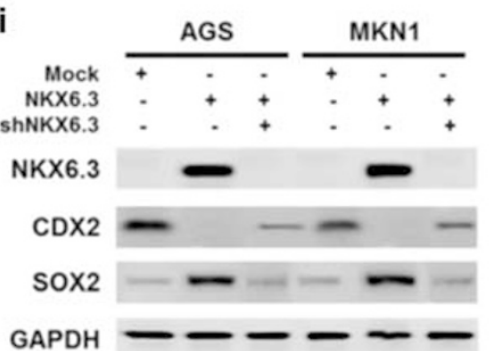

Figure 5 NKX6.3 functions as a transcriptional factor for $C D X 2$ and $S O X 2$ genes. (a and b) Putative promoter activity was characterized between $-6 \mathrm{~kb}$ and $+1 \mathrm{~kb}$ relative to the transcription start site of CDX2 and SOX2 genes using chromatin immunoprecipitation-PCR. NKX6.3-binding activity was detected in all fragments. (c and d) NKX6.3 occupancy was 7.2- and 4.75-fold enriched at the CDX2-transcription start site ${ }^{-5376}$ to 5215 and SOX2-transcription start site ${ }^{-4051}$ to 3838 compared with the control. TSS, transcription start site. (e and f) NKX6.3 downregulated CDX2 and Muc2 mRNA expression in both cells, but NKX6.3 silencing with shNKX6.3 completely reversed these effects. ( $\mathbf{g}$ and $\mathbf{h}$ ) NKX6.3 upregulated the expression of SOX2 and Muc5ac mRNAs, but NKX6.3 silencing with shNKX6.3 completely reversed these effects. (i) In AGS ${ }^{\mathrm{NKX} 6.3}$ and MKN1 ${ }^{\mathrm{NKX} 6.3}$ cells, NKX6.3 significantly downregulated CDX2 protein expression and upregulated SOX2 protein. 
$H$ \& $E$

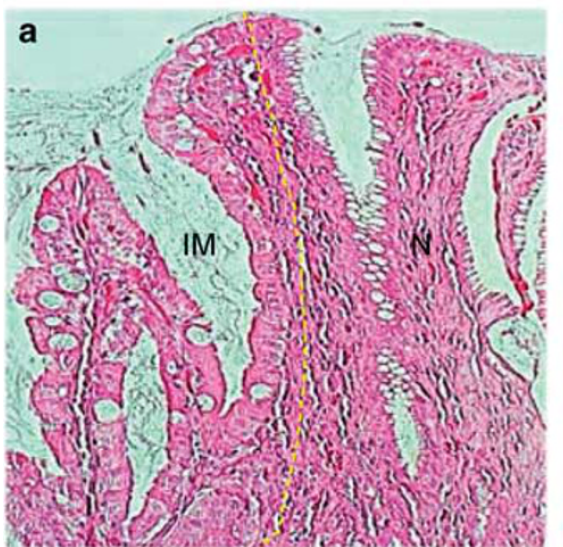

Muc5ac/DAPI

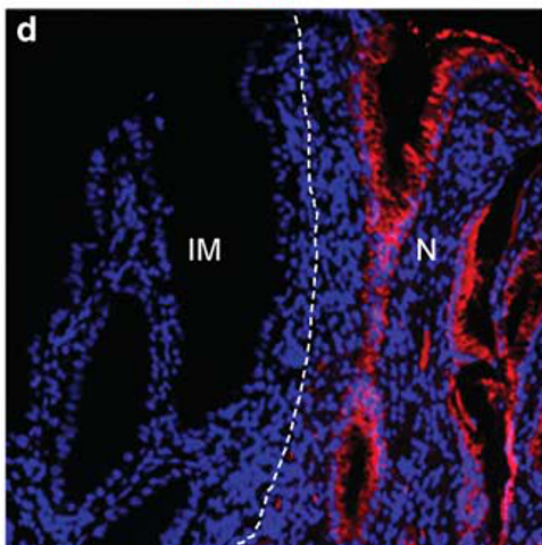

NKX6.3/GKN1/DAPI

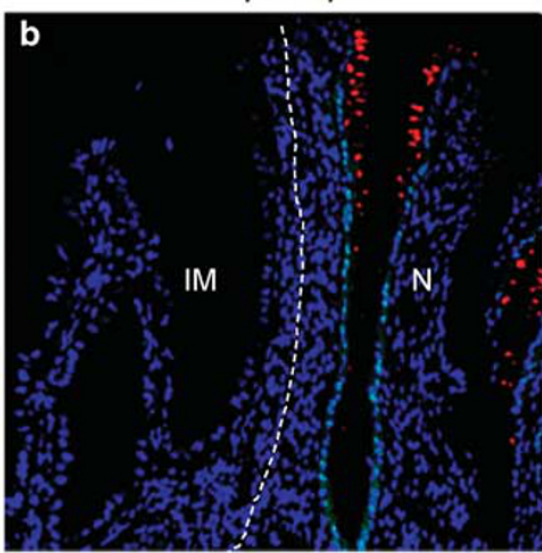

CDX2/DAPI

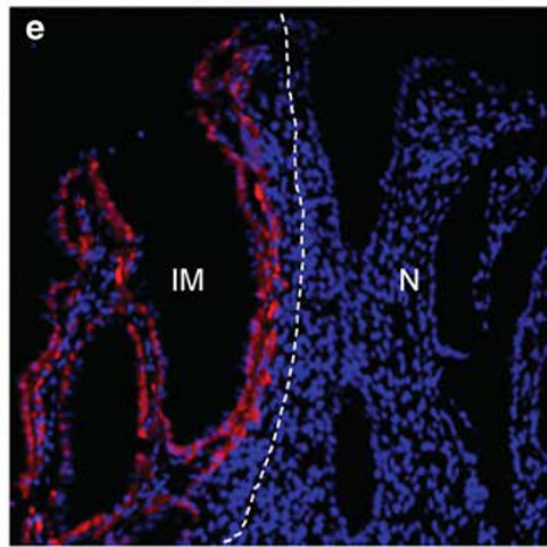

SOX2/DAP

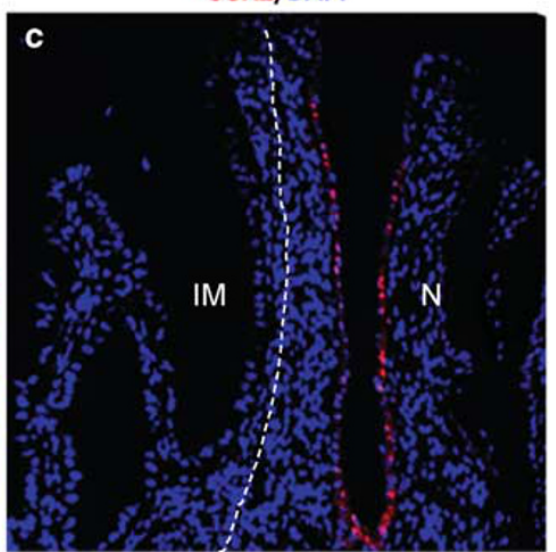

Muc2/DAPI

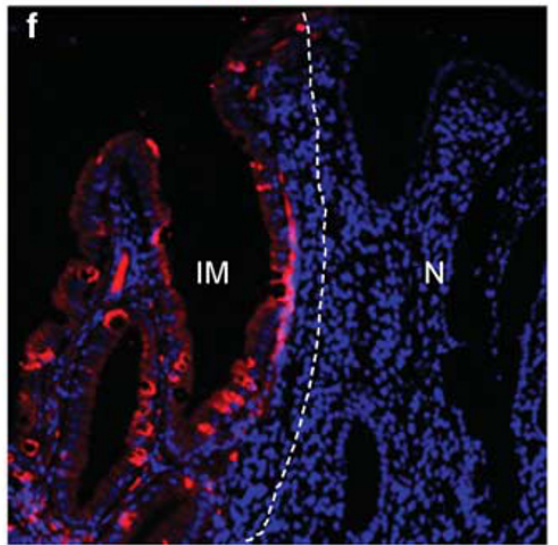

Figure 6 Expression of NKX6.3 target proteins in gastric mucosa. (a) Left half of gastric mucosa showed intestinal metaplasia (IM) containing goblet cells (H\&E staining). (b-f) In immunofluorescence analysis, nuclear localization of NKX6.3 protein was detected in gastric glandular epithelium without intestinal metaplasia (b). There was co-expression of NKX6.3, GKN1 (b), SOX2 (c), and Muc5ac (d) in gastric gland without intestinal metaplasia, whereas CDX2 (e) and Muc2 (f) expression was observed in gastric gland with intestinal metaplasia and NKX6.3-negativity. N, gastric gland without intestinal metaplasia.

that NKX6.3 may be a key factor for gastric differentiation.

The fact that NKX6.3 expression was positively correlated with SOX2 expression and inversely correlated with CDX2 in non-neoplastic gastric mucosal tissues implies that NKX6.3 regulates the expression of the gastric marker SOX2 and the intestinal marker CDX2. The promoter regions between $-6 \mathrm{~kb}$ and $+1 \mathrm{~kb}$ from the transcription start site of the SOX2 and CDX2 genes contain 29 and 12 putative NKX6.3binding motifs (TAAT), respectively. In chromatin immunoprecipitation-PCR analysis, NKX6.3 binds directly to promoter regions of the SOX2 and CDX2 genes and regulates their expression at mRNA and protein levels (Figures 5a-i). In addition, increased expression of Muc5ac and reduced Muc2 expression were also observed in $\mathrm{AGS}^{\mathrm{NKX} 6.3}$ and MKN1 ${ }^{\mathrm{NKX} 6.3}$ cells (Figures $5 \mathrm{~g}$ and $\mathrm{h}$ ), further confirming that SOX2 and CDX2 function as transcription factors for Muc5ac and Muc2, respectively. ${ }^{36,40,41}$ These results indicate that NKX6.3 may have an important role in gastric differentiation by acting as a transcriptional factor for $S O X 2$ and $C D X 2$ genes.
Next, to further confirm that NKX6.3 is a major regulator of gastric mucosal epithelium, we analyzed the expression of NKX6.3, GKN1, Muc5ac, CDX2, and Muc2 in human gastric mucosa by immunofluorescence assay. As seen in Figure 6, normal gastric glandular epithelium with NKX6.3 immunoreactivity showed SOX2, GKN1, and Muc5ac expression but not CDX2 and Muc2. In gastric mucosal glands with intestinal metaplasia, NKX6.3 expression was not observed and co-expression of both CDX2 and Muc2 proteins was markedly detected (Figure 6). Thus these results suggest that intestinal transformation of gastric epithelial cells may be a result of NKX6.3 inactivation through upregulation of CDX2, further supporting our in vitro data indicating that NKX6.3 may control gastric and intestinal differentiation.

It is well known that $H$. pylori is a causative agent of gastric cancer. ${ }^{47} \mathrm{H}$. pylori babA, cagA, and s1 vacA genes work synergistically to cause intestinal metaplasia ${ }^{48}$ which is an independent risk factor for gastric cancer development. ${ }^{49}$ In the present study, we examined the effects of $H$. pylori CagA on gastric 

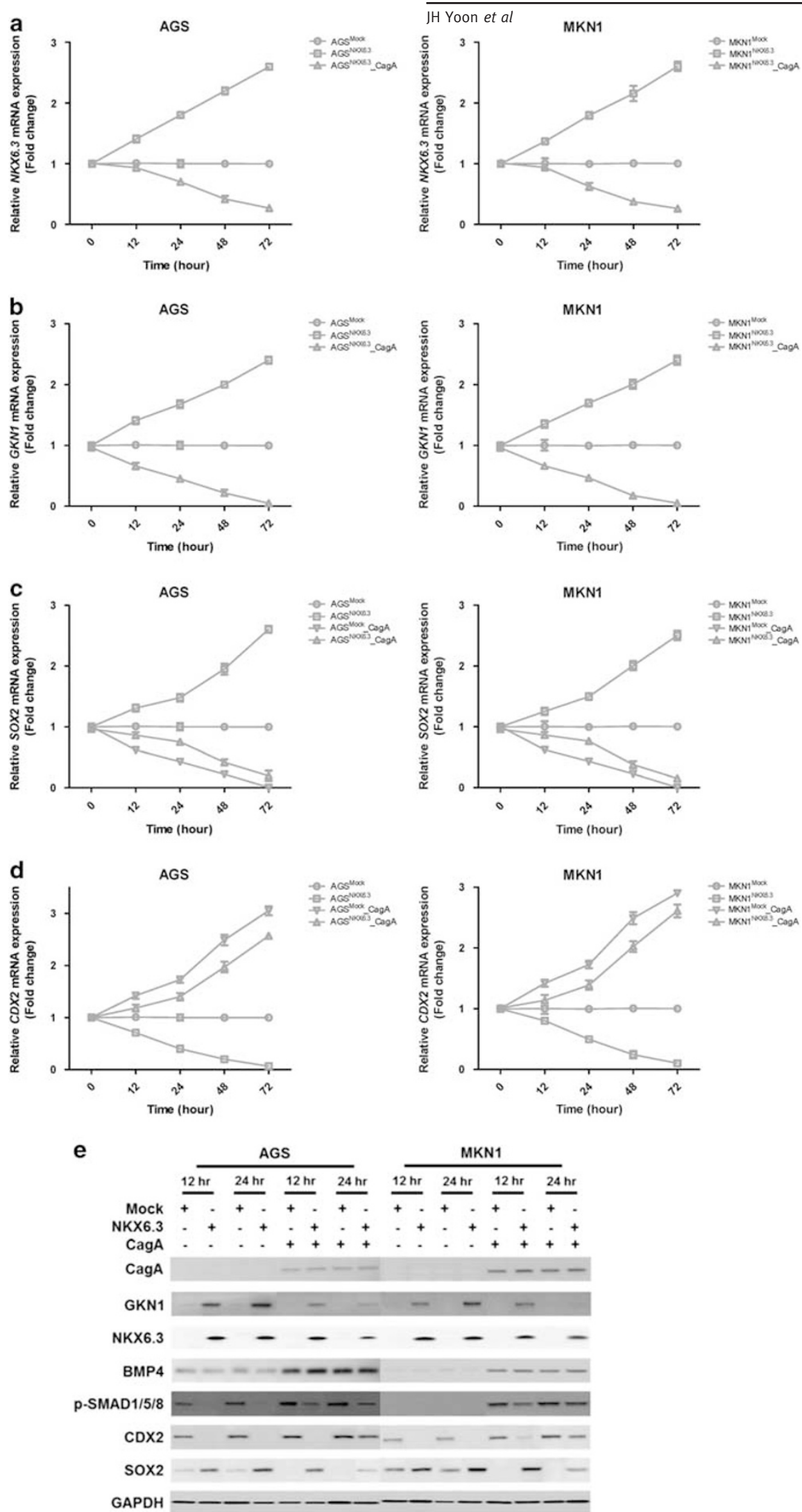

Figure $7 \mathrm{H}$. pylori CagA controls the expression of gastric and intestinal markers. (a) CagA-transfected AGS ${ }^{\mathrm{NKX} 6.3}$ and MKN1 ${ }^{\mathrm{NKX} 6.3}$ cells showed time-dependent inhibition of NKX6.3 expression. (b) Ectopic CagA expression downregulated GKN1 mRNA expression in both AGS $^{\text {NKX6.3 }}$ and MKN1 ${ }^{\text {NKX6.3 }}$ cells. (c) Ectopic CagA expression significantly inhibited SOX2 expression, even in NKX6.3 stable AGS ${ }^{\text {NKX6.3 }}$ and MKN1 ${ }^{\mathrm{NKX} 6.3}$ cells. (d) CagA-transfected AGS and MKN1 cells upregulated the expression of CDX2 mRNA, and stable NKX6.3 expression did not affect CagA-induced CDX2 upregulation. (e) CagA reduced NKX6.3, GKN1, and SOX2 protein expression and increased BMP4, CDX2, and p-SMAD1/5/8 expression in both AGS and MKN1 cells. 

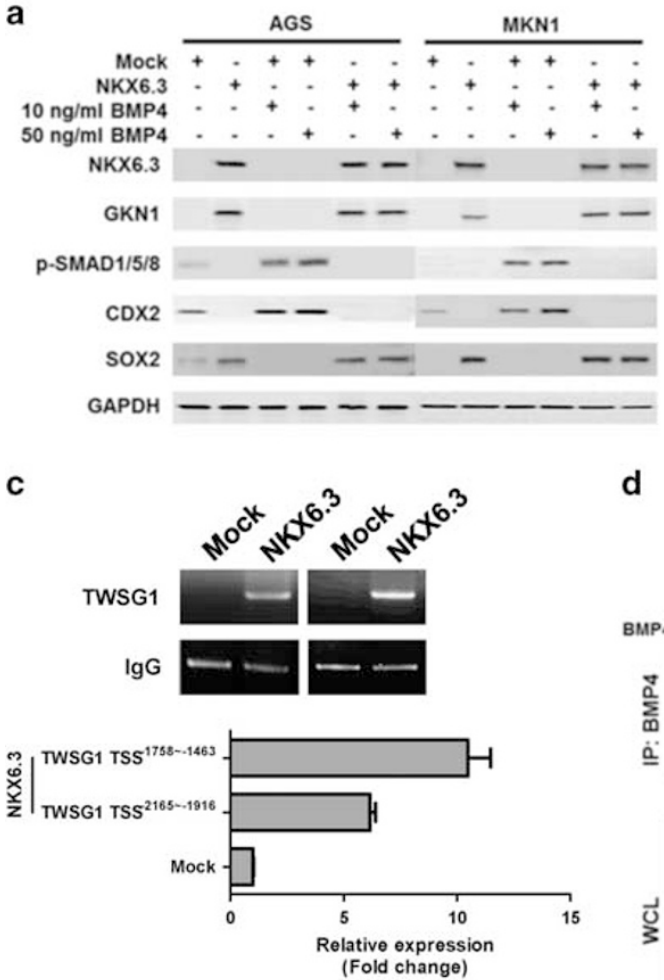

d b

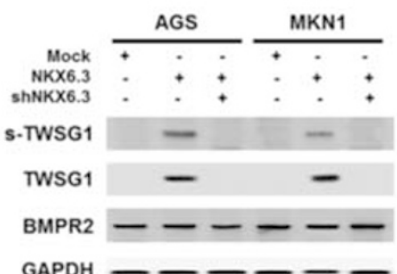

$\mathrm{GAPDH}---\ldots$

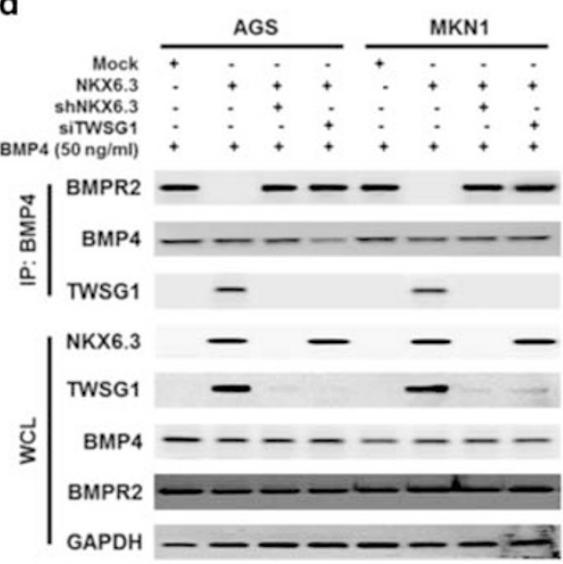

Figure 8 NKX6.3 inhibits BMP/p-SMAD signaling pathway. (a) Treatment of BMP4 in AGS and MKN1 cells induced the expression of p-SMAD1/5/8 and CDX2 proteins. However, BMP4 had no effect in NKX6.3 stable cells. (b) NKX6.3 induced secretary (s-TWSG1) and endogenous TWSG1 expression, but it did not affect BMPR-II expression. (c) Putative promoter activity was characterized between - $2.2 \mathrm{~kb}$ and $-1.4 \mathrm{~kb}$ relative to the transcription start site of TWSG1 genes by chromatin immunoprecipitation-PCR. NKX6.3 occupancy was 11.5- and 6.5-fold enriched at the TWSG1-transcription start site-1758 to -1463 and -transcription start site ${ }^{-2165}$ to - 1916 compared with control. TSS, transcription start site. (d) In BMP4-treated NKX6.3 stable cells, NKX6.3 inhibited BMP4-binding activity to BMPR-II protein by binding directly to BMP4. Immunoprecipitated proteins were resolved by SDS-PAGE, transferred to PVDF membranes and processed for immunoblotting with the indicated antibodies.

intestinal metaplasia in AGS and MKN1 gastric cancer cell lines and found that CagA reduced the expression of NKX6.3, GKN1, and SOX2 genes at mRNA and protein levels in a time-dependent manner (Figures 7a-e) but increased CDX2, BMP4, and p-SMAD1/5/8 expression in both AGS and MKN1 cells (Figures 7d and e). These results are in line with the report that $H$. pylori CagA increased CDX2 protein expression. ${ }^{23}$ Although NKX6.3 downregulated the expression of CDX2 and p-SMAD1/5/8 proteins, it did not affect BMP4 expression (Figure 7e). As reduced NKX6.3 DNA copy number as well as mRNA and protein expression were found in $\mathrm{CagA}(+) H$. pylori-infected gastric mucosae (Figure 3), we concluded that CagA $(+) H$. pylori infection leads to intestinal metaplasia by upregulating CDX2 and BMP4 expression through NKX6.3 inactivation.

Previously, it was shown that BMP2 and BMP4 regulate CDX2 expression and promote intestinal differentiation. ${ }^{24}$ In the present study, NKX6.3 did not affect BMP4 expression but reduced p-SMAD1/5/8 and CDX2 expression (Figure 7e). Next we analyzed the effects of BMP4 on NKX6.3 by examining the expression of NKX6.3, SOX2, CDX2, and p-SMAD1/5/8 after BMP4 treatment. Interestingly, treatment with BMP4 induced p-SMAD1/5/8 and CDX2 expression in AGS $^{\text {Mock }}$ and MKN1 ${ }^{\text {Mock }}$ cells, whereas NKX6.3 completely inhibited BMP4 activity in $\mathrm{AGS}^{\mathrm{NKX} 6.3}$ and MKN1 ${ }^{\mathrm{NKX6.3}}$ cells (Figure 8a). Thus we investigated whether NKX6.3 downregulates BMP4-induced CDX2 expression by inducing a cytokine named twisted gastrulation (TWSG1), which inhibits BMP-mediated phosphorylation of Smad proteins. ${ }^{27}$ Notably, NKX6.3 functioned as a transcriptional factor for the TWSG1 gene and increased its expression (Figures $8 \mathrm{~b}$ and c). Furthermore, NKX6.3-induced TWSG1 bound directly to BMP4 and inhibited BMP4-binding activity to BMPR-II (Figure 8d). These results suggest that NKX6.3 may prevent gastric intestinal metaplasia by inactivating the BMP/p-SMAD/CDX2 pathway through upregulating TWSG1 expression.

As GKN1 is a novel autocrine/paracrine protein specifically expressed in gastric mucosa and functions as a gastric specific tumor suppressor, ${ }^{20,50}$ we set out to determine whether NKX6.3 activity in gastric differentiation is dependent on GKN1. 
As shown in Supplementary Figure S2, NKX6.3 induced increased expression of GKN1 and SOX2 and decreased the expression of CDX2 at mRNA levels. In addition, GKN1 silencing with shGKN1 did not affect the expression of these genes, suggesting that NKX6.3 may control gastric differentiation in a GKN1-independent manner.

Here we present evidence that NKX6.3 may have an important role in the differentiation of gastric mucosal epithelium. NKX6.3 induces the expression of gastric differentiation markers such as GKN1, SOX2, and Muc5ac and inhibits the expression of intestinal differentiation markers, such as CDX2 and Muc2. Notably, NKX6.3 functions as a transcription factor for SOX2, CDX2, and TWSG1 genes by directly binding to their promoter regions. In immunofluorescence assay, NKX6.3 expression was not observed in gastric glands with intestinal metaplasia. Furthermore, NKX6.3-induced TWSG1 inhibited BMP4binding activity to BMPR-II by directly binding to BMP4. Therefore, we conclude that loss of NKX6.3, a key regulator of gastric differentiation, leads to transdifferentiation of gastric epithelial cells to an intestinal phenotype. It is plausible that modulating NKX6.3 activity could significantly impact the development of novel treatments, which will ultimately result in achieving the goal of gastric intestinal metaplasia recovery and gastric cancer prevention.

\section{Acknowledgments}

This work was supported by the Basic Science Research Program through the National Research Foundation of Korea (NRF) funded by the Ministry of Education, Science and Technology (2015R1A2A2A 05001023 and 2014R1A1A2058693). We thank Seong Yeob Ryu, Department of Gastroenterologic Surgery, Chonnam National University Hwasun Hospital, 160, Ilsim-ri, Hwasun-eup, Hwasun-gun, Jeollanam-do, 519-809, Korea for providing the gastric cancer samples with clinical information.

\section{Disclosure/conflict of interest}

The authors declare no conflict of interest.

\section{References}

1 Jermal A, Bray F, Center MM et al. Global cancer statistics. CA Cancer J Clin 2011;61:69-90.

2 Correa P. Human gastric carcinogenesis: a multistep and multifactorial process-First American Cancer Society Award Lecture on Cancer Epidemiology and Prevention. Cancer Res 1992;52:6735-6740.

3 Correa P, Haenszel W, Cuello C et al. A model for gastric cancer epidemiology. Lancet 1975;2:58-60.

4 Correa P, Piazuelo MB. The gastric precancerous cascade. J Dig Dis 2012;13:2-9.
5 Kneller RW, You WC, Chang YS et al. Cigarette smoking and other risk factors for progression of precancerous stomach lesions. J Natl Cancer Inst 1992;84:1261-1266.

6 Leung WK, Lin SR, Ching JY et al. Factors predicting progression of gastric intestinal metaplasia: results of a randomised trial on Helicobacter pylori eradication. Gut 2004;53:1244-1249.

7 Correa P, Haenszel W, Cuello C et al. Gastric precancerous process in a high risk population: crosssectional studies. Cancer Res 1990;50:4731-4736.

8 Filipe MI, Muñoz N, Matko I et al. Intestinal metaplasia types and the risk of gastric cancer: a cohort study in Slovenia. Int J Cancer 1994;57:324-329.

9 Reis CA, David L, Correa P et al. Intestinal metaplasia of human stomach displays distinct patterns of mucin (MUC1, MUC2, MUC5AC, and MUC6) expression. Cancer Res 1999;59:1003-1007.

10 Beck F, Chawengsaksophak K, Waring P et al. Reprogramming of intestinal differentiation and intercalary regeneration in Cdx2 mutant mice. Proc Natl Acad Sci USA 1999;96:7318-7323.

11 Silberg DG, Sullivan J, Kang E et al. Cdx2 ectopic expression induces gastric intestinal metaplasia in transgenic mice. Gastroenterology 2002;122: 689-696.

12 Rugge M, Ingravallo G, Farinati $\mathrm{F}$ et al. Re: CDX2 homeotic gene expression in gastric noninvasive neoplasia. Am J Surg Pathol 2004;28:834-835.

13 Uemura N, Okamoto S, Yamamoto S et al. Helicobacter pylori infection and the development of gastric cancer. N Engl J Med 2001;345:784-789.

14 Choi MY, Romer AI, Wang Y et al. Requirement of the tissue-restricted homeodomain transcription factor Nkx6.3 in differentiation of gastrin-producing G cells in the stomach antrum. Mol Cell Biol 2008;28: 3208-3218.

15 Alanentalo T, Chatonnet F, Karlen $\mathrm{M}$ et al. Cloning and analysis of Nkx6.3 during CNS and gastrointestinal development. Gene Expr Patterns 2006;6:162-170.

16 Karam SM, Leblond CP. Identifying and counting epithelial cell types in the "corpus" of the mouse stomach. Anat Rec 1992;232:231-246.

17 Yoon JH, Choi WS, Kim O et al. NKX6.3 induces gastric differentiation and inhibits gastric carcinogenesis. Oncotarget 2015;6:28425-28439.

18 Dixon MF, Genta RM, Yardley JH et al. Classification and grading of gastritis. The updated Sydney System. International Workshop on the Histopathology of Gastritis, Houston 1994. Am J Surg Pathol 1996;20: 1161-1181.

19 Yoon JH, Seo HS, Choi SS et al. Gastrokine 1 inhibits the carcinogenic potentials of Helicobacter pylori CagA. Carcinogenesis 2014;35:2619-2629.

20 Yoon JH, Song JH, Zhang C et al. Inactivation of the Gastrokine 1 gene in gastric adenomas and carcinomas. J Pathol 2011;223:618-625.

21 Lee A, O'Rourke J, De Ungria MC et al. A standardized mouse model of Helicobacter pylori infection: introducing the Sydney strain. Gastroenterology 1997;112: 1386-1397.

22 Choi WS, Seo HS, Song KY et al. Gastrokine 1 expression in the human gastric mucosa is closely associated with the degree of gastritis and DNA methylation. J Gastric Cancer 2013;13:232-241.

23 Song X, Chen HX, Wang XY et al. H. pylori-encoded CagA disrupts tight junctions and induces invasiveness 
of AGS gastric carcinoma cells via Cdx2-dependent targeting of Claudin-2. Cell Immunol 2013;286:22-30.

24 Barros R, Pereira B, Duluc I et al. Key elements of the $\mathrm{BMP} / \mathrm{SMAD}$ pathway co-localize with CDX2 in intestinal metaplasia and regulate CDX2 expression in human gastric cell lines. J Pathol 2008;215:411-420.

25 Mari L, Milano F, Parikh $\mathrm{K}$ et al. A pSMAD/CDX2 complex is essential for the intestinalization of epithelial metaplasia. Cell Rep 2014;7:1197-1210.

26 Pham L, Beyer K, Jensen ED et al. Bone morphogenetic protein 2 signaling in osteoclasts is negatively regulated by the BMP antagonist, twisted gastrulation. J Cell Biochem 2011;112:793-803.

27 Tanno T, Porayette P, Sripichai O et al. Identification of TWSG1 as a second novel erythroid regulator of hepcidin expression in murine and human cells. Blood 2009;114:181-186.

28 Khurana S, Mills JC. The gastric mucosa development and differentiation. Prog Mol Biol Transl Sci 2010;96: 93-115.

29 Karam SM, Leblond CP. Dynamics of epithelial cells in the corpus of the mouse stomach. III. Inward migration of neck cells followed by progressive transformation into zymogenic cells. Anat Rec 1993;236:297-313.

30 Fukaya M, Isohata N, Ohta $\mathrm{H}$ et al. Hedgehog signal activation in gastric pit cell and in diffuse-type gastric cancer. Gastroenterology 2006;131:14-29.

31 Nomura S, Settle SH, Leys CM et al. Evidence for repatterning of the gastric fundic epithelium associated with Ménétrier's disease and TGFalpha overexpression. Gastroenterology 2005;128:1292-1305.

32 Almeida R, Almeida J, Shoshkes M et al. OCT-1 is overexpressed in intestinal metaplasia and intestinal gastric carcinomas and binds to, but does not transactivate, CDX2 in gastric cells. J Pathol 2005;207:396-401.

33 Que J, Okubo T, Goldenring JR et al. Multiple dosedependent roles for Sox2 in the patterning and differentiation of anterior foregut endoderm. Development 2007;134:2521-2531.

34 Ishii $\mathrm{Y}$, Rex M, Scotting PJ et al. Region-specific expression of chicken Sox2 in the developing gut and lung epithelium: regulation by epithelial-mesenchymal interactions. Dev Dyn 1998;213:464-475.

35 Tani Y, Akiyama Y, Fukamachi $\mathrm{H}$ et al. Transcription factor SOX2 up-regulates stomach-specific pepsinogen A gene expression. J Cancer Res Clin Oncol 2007;133: 263-269.

$36 \mathrm{Li}$ XL, Eishi Y, Bai YQ et al. Expression of the SRYrelated HMG box protein in human gastric carcinoma. Int J Oncol 2004;24:257-263.

37 Tsukamoto $\mathrm{T}$, Inada $\mathrm{K}$, Tanaka $\mathrm{H}$ et al. Down regulation of a gastric transcription factor, Sox2, and ectopic expression of intestinal homeobox genes, Cdx1 and Cdx2: inverse correlation during progression from gastric/intestinal-mixed to complete intestinal metaplasia. J Cancer Res Clin Oncol 2004;130:135-145.

38 Otsubo T, Akiyama Y, Yanagihara K et al. SOX2 is frequently downregulated in gastric cancers and inhibits cell growth through cell-cycle arrest and apoptosis. Br J Cancer 2008;98:824-831.

39 Young T, Rowland JE, van de Ven C et al. Cdx and Hox genes differentially regulate posterior axial growth in mammalian embryos. Dev Cell 2009;17:516-526.

40 Almeida R, Silva E, Santos-Silva F et al. Expression of intestine-specific transcription factors, CDX1 and CDX2, in intestinal metaplasia and gastric carcinomas. J Pathol 2003;199:36-40.

41 Yamamoto H, Bai YQ, Yuasa Y. Homeodomain protein CDX2 regulates goblet-specific MUC2 gene expression. Biochem Biophys Res Commun 2003;300: 813-818.

42 Liu Q, Teh $\mathrm{M}$, Ito $\mathrm{K}$ et al. CDX2 expression is progressively decreased in human gastric intestinal metaplasia, dysplasia and cancer. Mod Pathol 2007;20: 1286-1297.

43 Obst B, Wagner S, Sewing KF et al. Helicobacter pylori causes DNA damage in gastric epithelial cells. Carcinogenesis 2000;21:1111-1115.

$44 \mathrm{Wu}$ CW, Chen GD, Fann CS et al. Clinical implications of chromosomal abnormalities in gastric adenocarcinomas. Genes Chromosomes Cancer 2002;35:219-231.

45 Cobler L, Pera M, Garrido $\mathrm{M}$ et al. CDX2 can be regulated through the signalling pathways activated by IL-6 in gastric cells. Biochim Biophys Acta 2014;1839: 785-792.

46 Simmini S, Bialecka M, Huch M et al. Transformation of intestinal stem cells into gastric stem cells on loss of transcription factor Cdx2. Nat Commun 2014;5:5728.

47 Blaser MJ, Perez-Perez GI, Kleanthous H et al. Infection with Helicobacter pylori strains possessing cagA is associated with an increased risk of developing adenocarcinoma of the stomach. Cancer Res 1995;55: 2111-2115.

48 Zambon CF, Navaglia F, Basso D et al. Helicobacter pylori babA2, cagA, and s1 vacA genes work synergistically in causing intestinal metaplasia. J Clin Pathol 2003;56:287-291.

49 Cassaro M, Rugge M, Gutierrez O et al. Topographic patterns of intestinal metaplasia and gastric cancer. Am J Gastroenterol 2000;95:1431-1438.

50 Martin TE, Powell CT, Wang Z et al. A novel mitogenic protein that is highly expressed in cells of the gastric antrum mucosa. Am J Physiol Gastrointest Liver Physiol 2003;285:G332-G343.

Supplementary Information accompanies the paper on Modern Pathology website (http://www.nature.com/ modpathol) 